\title{
Gamma Rays from SN 1987A due to Pseudoscalar Conversion
}

\author{
J. A. Grifols, E. Massó, and R. Toldrà \\ Grup de Física Teòrica and IFAE, Edifici Cn, Universitat Autònoma de Barcelona, E-08193 Bellaterra, Spain
} (Received 9 May 1996)

\begin{abstract}
A light pseudoscalar coupled to two photons would be copiously emitted by the core of a supernova. Part of this flux would be converted to $\gamma$ rays by the galactic magnetic field. Measurements on the SN 1987A $\gamma$-ray flux by the Gamma-Ray Spectrometer on the Solar Maximum Mission satellite already imply a bound on the coupling $g<3 \times 10^{-12} \mathrm{GeV}^{-1}$. The improved generation of satellite-borne detectors, like EGRET or the project GLAST, could be able to detect a pseudoscalar-to-photon signal from a nearby supernova, for allowed values of $g$. [S0031-9007(96)01219-7]
\end{abstract}

PACS numbers: 95.30.Cq, 14.80.Mz, 95.85.Pw, 97.60.Bw

Pseudoscalar particles are often fundamental ingredients of particle physics models. Examples are axions [1] or Majorons [2], coming from models with spontaneous breaking of a Peccei-Quinn symmetry [3] or of a global lepton symmetry, respectively. Other examples are light bosons from extra-dimensional gauge theories [4], arions [5], and omions [6]. Pseudoscalar particles usually couple to two photons via the interaction Lagrangian

$$
\mathcal{L}=\frac{1}{8} g \phi \varepsilon_{\mu \nu \alpha \beta} F^{\mu \nu} F^{\alpha \beta} .
$$

Limits on the coupling $g$ come from laboratory experiments and from cosmological and astrophysical observations. These have been discussed and collected in [7]. Further astrophysical constraints have been recently examined by Mori [8]. The limits on $g$ depend on the mass $m$ of the pseudoscalar. For very light masses, $m \leq$ $10^{-9} \mathrm{eV}$, the best constraints come from astrophysics (such constraints are not interesting for axion models, where $g$ and $m$ are related; but are of interest to other models where $m \leq 10^{-9} \mathrm{eV}$ does not imply an exceedingly small coupling $g$ ). Mohanty and Nayak [9] considered the creation of a $\phi$ background due to strong magnetic fields in pulsars. Pulsar signals propagating through this background show a time lag between different modes of polarization. They found the limit

$$
g \leq 5.3 \times 10^{-11} \mathrm{GeV}^{-1},
$$

valid for $m \leq 10^{-10} \mathrm{eV}$. Another way to constrain $g$ for $m \leq 10^{-9} \mathrm{eV}$ has been discussed by Carlson [10]. He studied $\mathrm{x}$-ray conversion of $\phi$ produced in giant cores and found the following limit:

$$
g \leq 2.5 \times 10^{-11} \mathrm{GeV}^{-1},
$$

using HEAO1 satellite data on $\alpha$-Ori x-ray emission. A stringent bound on $g$ has been recently obtained by Krasnikov [11]: $\phi \rightarrow \gamma$ conversion would lead to a large scale anisotropy of the x-ray background. The observed isotropy leads to the estimation [11]

$$
g \leq 1 \times 10^{-11} \mathrm{GeV}^{-1} .
$$

This author also considers similar effects to those studied by Carlson [10]. In addition, he discusses po- larimetric effects in the emission by magnetic white dwarfs, and claims measurements may be sensitive to $g \sim 10^{-11} \mathrm{GeV}^{-11}$.

In the present paper, we show that it is possible to improve these bounds on $g$ for $m \leq 10^{-9} \mathrm{eV}$. We will use the fact that, should these pseudoscalars exist, they are copiously produced when a supernova occurs. For small $g$, these particles will stream away from the supernova core, without further interactions. In their path, the galactic magnetic field will convert a fraction of the flux back to gamma-ray photons. At the time the neutrino burst from SN 1987A was observed, the GammaRay Spectrometer (GRS) on the Solar Maximum Mission (SMM) satellite was operative and did not observe such a gamma-ray signal. We use this null result to set the stringent limit

$$
g<3 \times 10^{-12} \mathrm{GeV}^{-1} .
$$

The next generation of detectors, like EGRET on the Compton GRO satellite or the project GLAST, could be able to detect a $\gamma$-ray signal due to $\phi$ conversion from a nearby supernova collapse, for values of $g$ allowed by our analysis.

Pseudoscalar production. - Immediately after collapse, $\phi$ is produced in the hot and superdense core of the supernova. In what follows, we take a core temperature $T=60 \mathrm{MeV}$, proton number density $n_{p}=1.4 \times 10^{38} \mathrm{~cm}^{-3}$, and radius $R_{c}=10 \mathrm{~km}$. As has been discussed in [12], a source of uncertainty arises because we do not know which is the equation of state at supernuclear densities. To estimate the uncertainty, we will follow that reference and change the central density by a factor of 2 . All the other parameters will change correspondingly. For example, the core temperature changes by a factor of 1.6, if one assumes an isentropic collapse. Notice that this allows the core temperature to span the conventional range of $T \sim 30-100 \mathrm{MeV}$. Since $\phi$ couples to the electromagnetic field, the relevant interactions will involve electrons and protons. The Fermi momentum of electrons and protons is $p_{F}=320 \mathrm{MeV}$. For the relativistic electrons, $p_{F} / T \gg 1$, thus they are extremely degenerate. As to 
the nonrelativistic protons, $\left(p_{F}^{2} / 2 m_{p}\right) /(3 T / 2) \simeq 0.6$, and hence they are only moderately degenerate.

Let us start considering the Primakoff process on protons, $p \gamma \rightarrow p \phi$, that leads to $\phi$ creation. The number of pseudoscalars produced per unit volume and per unit time, at temperature $T$ and with energy between $E_{\min }$ and $E_{\max }$ is

$$
N(T)=\int_{E_{\min }}^{E_{\max }} \sigma(\omega) v n_{p} d n_{\gamma}(T, \omega)
$$

We integrate between $E_{\min }$ and $E_{\max }$ since gamma-ray detectors are only sensitive to a fixed energy band. In expression (6) $n_{p}$ and $n_{\gamma}$ are the number densities of protons and photons, respectively, $v$ their relative velocity, and the Primakoff cross section as a function of the photon energy $\omega$ is (see [13])

$$
\sigma(\omega)=\frac{1}{8} \alpha g^{2}\left[\left(1+\frac{\kappa^{2}}{4 \omega^{2}}\right) \ln \left(1+\frac{4 \omega^{2}}{\kappa^{2}}\right)-1\right] .
$$

The screening wave number $\kappa$ appears because of the collective behavior of the plasma, which cuts off the range of the Coulomb potential for scales larger than $\sim \kappa^{-1}$. It is given by

$$
\begin{gathered}
\kappa^{2}=\kappa_{D}^{2}+\kappa_{T F}^{2}, \\
\kappa_{D}^{2}=\frac{4 \pi \alpha n_{p}}{T}, \\
\kappa_{T F}^{2}=\frac{4 \alpha}{\pi} p_{F} E_{F},
\end{gathered}
$$

with $p_{F}$ and $E_{F}$ the Fermi momentum and energy of the electrons. The proton contribution in our case is $\kappa_{D}=50 \mathrm{MeV}$, where we are considering the protons not degenerate (see below). The contribution of the degenerate electrons is given by $\kappa_{T F}=33 \mathrm{MeV}$, smaller than the proton contribution. Protons can move in the plasma (or, better said, in momentum space) more freely than the degenerate electrons, and so they can screen charges more easily than the electrons.

In order to obtain $\sigma(\omega)$ in Eq. (7) one needs to know the thermal average $\left\langle(F(q))^{2}\right\rangle$, being $F(q)$ the usual form factor associated with the charge distribution of the plasma. To make the thermal average we need $p_{i j}^{Q}(r)$, the probability per unit volume of finding the charge $Z_{j}$ at a distance $r$ of the charge $Z_{i}[13]$

$$
p_{i j}^{Q}(r)=\frac{1}{V}\left(1-\frac{Z_{i} Z_{j} \alpha}{T} \frac{\exp (-\kappa r)}{r}\right)
$$

for an arbitrarily large volume $V$. In addition to this charge correlation, one also has to consider the statistical correlation between fermions. The probability $p_{i j}^{S}(r)$ of finding a fermion $j$ at a distance $r$ of the fermion $i$ is [14]

$$
p_{i j}^{S}(r)=\frac{1}{V}\left[1-\exp \left(-m T r^{2}\right)\right] .
$$

One may estimate the relative importance of the two different types of correlation by evaluating the quotient

$$
\epsilon \equiv \frac{V^{-1}-p_{i j}^{S}(r)}{V^{-1}-p_{i j}^{Q}(r)} \quad \text { at } r=\kappa^{-1} .
$$

Using the physical parameters in the supernova core we obtain $\epsilon<10^{-5}$ for the gas of protons. Therefore we can neglect the near degeneracy of protons and consider only the charge correlation. In this case, one gets expression (9).

One might also consider other mechanisms of pseudoscalar production such as the Primakoff process on electrons, $e \gamma \rightarrow e \phi$, or processes involving only nucleons in the initial state, $p n \rightarrow p n \gamma \phi$, where a virtual photon attached either to a proton or to a virtual charged pion splits into the final $\gamma$ and $\phi$. These processes would add to the one previously considered and would make our limit on $g$ more stringent. However, the process $e \gamma \rightarrow e \phi$ is suppressed compared to $p \gamma \rightarrow p \phi$ due to the extreme degeneracy of the electrons; production of a pseudoscalar entails a change of momentum $\Delta p_{e} \sim T$ which is not allowed for the bulk of electrons in the Fermi sea (one has to bear in mind that forward peaks, in which $\Delta p_{e} \approx 0$, are suppressed by the finite range of the Coulomb potential in a plasma). Processes like $p n \rightarrow p n \gamma \phi$ are also unimportant since they can be visualized as $\gamma \phi$ production by the nearly static electric field created by protons. This sort of production is clearly diminished by energy conservation.

Finally, we can write the expression for the pseudoscalar flux $\Phi_{\phi}$ on the Earth, at a distance $D=55 \mathrm{kpc}$ from SN 1987A, considering $p \gamma \rightarrow p \phi$ as the only production mechanism in the supernova core,

$$
\begin{aligned}
\Phi_{\phi}= & 3 \times 10^{4} \mathrm{~cm}^{-2} \mathrm{~s}^{-1}\left(\frac{g}{10^{-11} \mathrm{GeV}^{-1}}\right)^{2}\left(\frac{55 \mathrm{kpc}}{D}\right)^{2} \\
& \times\left(\frac{R_{c}}{10 \mathrm{~km}}\right)^{3}\left(\frac{n_{p}}{1.4 \times 10^{38} \mathrm{~cm}^{-3}}\right)\left(\frac{T}{60 \mathrm{MeV}}\right)^{3} \\
& \times f\left(\xi^{2}, E_{\min }, E_{\max }\right),
\end{aligned}
$$

where

$$
\begin{aligned}
f\left(\xi^{2}, E_{\min }, E_{\max }\right)= & \frac{1}{2 \pi} \int_{x_{\min }}^{x_{\max }} d x \frac{1}{e^{x}-1} \\
& \times\left[\left(x^{2}+\xi^{2}\right) \ln \left(1+x^{2} / \xi^{2}\right)-x^{2}\right],
\end{aligned}
$$

and being

$$
\begin{gathered}
\xi=\kappa / 2 T, \\
x_{\min }=E_{\min } / T, \\
x_{\max }=E_{\max } / T .
\end{gathered}
$$

One can check that the energy drain by these pseudoscalars during the collapse of the supernova core is at least a factor of $10^{-4}$ smaller than the energy released in neutrinos; therefore, we can ignore the backreaction caused by $\phi$ emission on the supernova evolution. 
Pseudoscalar conversion. - The mixing between the photon and low mass particles in magnetic fields leads to very interesting phenomena. Since the pioneering work in [6,15], a variety of implications for laboratory experiments and astrophysical observations have been investigated (see references in [7]).

For our purposes, we use the formalism developed in [16]. The $\phi \rightarrow \gamma$ transition probability, for a beam traversing a transverse magnetic field $B_{T}$ after a distance $L$, is given by

$$
P(\phi \rightarrow \gamma)=\frac{1}{4} g^{2} B_{T}^{2} L^{2}\left(\frac{\sin x}{x}\right)^{2},
$$

with

$$
x=\frac{L}{2} \sqrt{\Delta_{\mathrm{osc}}^{2}+g^{2} B_{T}^{2}}
$$

and

$$
\Delta_{\mathrm{osc}}=\frac{\left|\omega_{p}^{2}-m^{2}\right|}{2 \omega},
$$

where $\omega$ is the energy and

$$
\omega_{p}^{2}=\frac{4 \pi \alpha n_{e}}{m_{e}}=\left(6.4 \times 10^{-12} \mathrm{eV}\right)^{2}\left(\frac{n_{e}}{0.03 \mathrm{~cm}^{-3}}\right)
$$

is the plasmon mass. We have normalized it to the mean electron density in the interstellar medium, $n_{e} \simeq$ $0.03 \mathrm{~cm}^{3}$.

A coherent effect, which implies $\sin x / x \rightarrow 1$, is obtained provided

$$
m \leq 10^{-9} \mathrm{eV},
$$

so that our constraint on $g$ will be valid only for such small masses. In order to evaluate the probability in (19), we need to specify the magnetic field structure. We will adopt the model used in [10], consisting of a toroidal $2 \mu \mathrm{G}$ magnetic field. The coherence length is about the order of several kiloparsecs [17]. To be conservative we take a coherence length $L=1 \mathrm{kpc}$. In the direction of SN 1987A one has

$$
B_{T}=(2 \mu \mathrm{G})\left(1-\sin ^{2} l \cos ^{2} b\right)^{1 / 2} \simeq 1 \mu \mathrm{G},
$$

where the corresponding galactic coordinates have been used.

Summing up, we have

$$
\begin{aligned}
P(\phi \rightarrow \gamma)= & 3 \times 10^{-3}\left(\frac{g}{10^{-11} \mathrm{GeV}^{-1}}\right)^{2} \\
& \times\left(\frac{B_{T}}{1 \mu \mathrm{G}}\right)^{2}\left(\frac{L}{1 \mathrm{kpc}}\right)^{2} .
\end{aligned}
$$

At the time the neutrino burst from SN 1987A reached the Earth, the satellite-borne GRS was on duty measuring the incident $\gamma$-ray flux. This measurement provides an observational limit on the $\gamma$-ray flux coming from the supernova, and consequently on the photons from supernova $\phi$ emission. Indeed, data from SMM [18] in the energy band $E_{\min }=25 \mathrm{MeV}<E<E_{\max }=100 \mathrm{MeV}$ imply that

$$
\Phi_{\phi} P(\phi \rightarrow \gamma) \Delta t<0.6 \mathrm{~cm}^{-2} .
$$

The characteristic time over which the protoneutron star emits most of its gravitational energy and therefore the bulk of the hypothetical $\phi$ particles is the diffusion time of neutrinos. Detailed stellar evolution calculations [19] render about $10 \mathrm{~s}$ for this diffusion time scale. To be conservative we shall take $\Delta t=5 \mathrm{~s}$ which is roughly the characteristic signal decay time for the neutrino burst of SN 1987A. We thus find the limit

$$
g<3 \times 10^{-12} \mathrm{GeV}^{-1} .
$$

Changing the physical parameters of the supernova as previously discussed, we estimate an uncertainty factor of 2 in the limit (27).

Finally, we would like to make some comments on the energies of the photons from pseudoscalar conversion. The expected spectrum has a mean energy value slightly bigger than $2.7 T \sim 160 \mathrm{MeV}$ (the energy spectrum is not exactly that of a black body since the Primakoff cross section increases with the energy). Thus, it extends above the GRS cutoff at $E_{\max }=100 \mathrm{MeV}$ and, consequently, part of it would not have been detected. Better prospects could be expected with EGRET on the Compton GRO satellite, launched in 1991, since it is able to detect $\gamma$ rays from $E_{\min }=20 \mathrm{MeV}$ up to $E_{\max }=30 \mathrm{GeV}$ and therefore is sensitive to the whole spectrum. In addition, the EGRET detector is more sensitive than the GRS detector. A pseudoscalar with $g$ allowed by Eq. (27) would possibly give a $\gamma$-ray signal in EGRET from a nearby supernova. Even more promising is the project GLAST with detectors to measure $\gamma$ rays in the energy range from $E_{\min }=50 \mathrm{MeV}$ to $E_{\max }=100 \mathrm{GeV}$, and a factor of 100 better in sensitivity than EGRET.

We thank the Theoretical Astroparticle Network for support under the EEC Contract No. CHRX-CT93-0120 (Direction Generale 12 COMA). This work has been partially supported by the CICYT Research Projects No. AEN95-0815 and No. AEN95-0882. R.T. acknowledges a FPI grant from the Ministerio de Educación y Ciencia (Spain).

Note added. - After submission of this paper, a preprint by Brockway, Carlson, and Raffelt [20] appeared. This preprint tackles the same problem we have studied in our paper and reaches basically the same results.

[1] S. Weinberg, Phys. Rev. Lett. 40, 223 (1978); F. Wilczek, Phys. Rev. Lett. 40, 279 (1978).

[2] Y. Chicashige, R. N. Mohapatra, and R. D. Peccei, Phys. Rev. Lett. 45, 1926 (1980); Phys. Lett. 98B, 265 (1981); G. Gelmini and M. Roncadelli, Phys. Lett. 99B, 411 (1981).

[3] R. D. Peccei and H. Quinn, Phys. Rev. Lett. 38, 1440 (1977); Phys. Rev. D 16, 1791 (1977). 
[4] N. Turok, Phys. Rev. Lett. 76, 1015 (1996).

[5] A. A. Anselm and N. G. Uraltsev, Phys. Lett. 114B, 39 (1982).

[6] P. Sikivie, Phys. Rev. Lett. 61, 783 (1988).

[7] E. Massó and R. Toldrà, Phys. Rev. D 52, 1755 (1995).

[8] F. Mori, Mod. Phys. Lett. A 11, 715 (1996).

[9] S. Mohanty and S. N. Nayak, Phys. Rev. Lett. 70, 4038 (1993); 76, 2825(E) (1996).

[10] E. D. Carlson, Phys. Lett. B 344, 245 (1995).

[11] S. V. Krasnikov, Phys. Rev. Lett. 76, 2633 (1996).

[12] M. S. Turner, Phys. Rev. Lett. 60, 1797 (1988).

[13] G. G. Raffelt, Phys. Rev. D 33, 897 (1986).

[14] R. Pathria, Statistical Mechanics (Oxford Pergamon Press,
New York, 1972).

[15] P. Sikivie, Phys. Rev. Lett. 51, 1415 (1983); Phys. Rev. D 32, 2988 (1985).

[16] G. Raffelt and L. Stodolsky, Phys. Rev. D 37, 1237 (1988).

[17] Y. A. Zeldovich, A. A. Ruzmaikin, and D. D. Sokoloff, Magnetic Fields in Astrophysics (Gordon and Breach Science Publishers, New York, 1983).

[18] E. L. Chupp, W. T. Vestrand, and C. Reppin, Phys. Rev. Lett. 62, 505 (1989).

[19] A. Burrows, Annu. Rev. Nucl. Part. Sci. 40, 181 (1990).

[20] J. W. Brockway, E. D. Carlson, and G. G. Raffelt, Report No. astro-ph/9605197 [Phys. Lett. B (to be published)]. 